\title{
On a Rigidity Problem of Beardon and Minda
}

\author{
Baokui $\mathrm{Li}^{1,2} \cdot$ Yuefei Wang ${ }^{3,4}$
}

Received: 20 September 2020 / Revised: 11 February 2021 / Accepted: 29 March 2021 /

Published online: 17 June 2021

(c) The Author(s) 2021

\section{Abstract}

In this paper, we give a positive answer to a rigidity problem of maps on the Riemann sphere related to cross-ratios, posed by Beardon and Minda (Proc Am Math Soc 130(4):987-998, 2001). Our main results are: (I) Let $E \not \subset \hat{\mathbb{R}}$ be an arc or a circle. If a map $f: \hat{\mathbb{C}} \mapsto \widehat{\mathbb{C}}$ preserves cross-ratios in $E$, then $f$ is a Möbius transformation when $\bar{E} \neq E$ and $f$ is a Möbius or conjugate Möbius transformation when $\bar{E}=E$, where $\bar{E}=\{\bar{z} \mid z \in E\}$. (II) Let $E \subset \hat{\mathbb{R}}$ be an arc satisfying the condition that the cardinal number $\#(E \cap\{0,1, \infty\})<2$. If $f$ preserves cross-ratios in $E$, then $f$ is a Möbius or conjugate Möbius transformation. Examples are provided to show that the assumption $\#(E \cap\{0,1, \infty\})<2$ is necessary.

Keywords Cross-ratios · Absolute cross-ratios · Möbius transformations · Conjugate Möbius transformations

Mathematics Subject Classification 51F15 · 30C35

In memory of Professor Walter Hayman.

Communicated by James K. Langley.

Yuefei Wang was supported by NSF of China (No. 11688101).

$凶$ Yuefei Wang

wangyf@math.ac.cn

Baokui Li

henan_lbk@bit.edu.cn

1 School of Mathematics and Statistics, Beijing Institute of technology, Beijing 100081, China

2 Beijing Key Laboratory on MCAACI, Beijing Institute of technology, Beijing 100081, China

3 College of Mathematics and Statistics, Shenzhen University, Shenzhen 518060, Guandong, China

4 Institute of Mathematics, Academy of Mathemtics and Systems Sciences, Chinese Academy of Sciences, Beijing 100190, China 


\section{Introduction and Main Results}

In this paper we consider the relation between Möbius transformations and crossratios of points in the complex plane. For any four distinct points $a, b, c$, and $d$ in the extended complex plane $\hat{\mathbb{C}}$, their cross-ratio and absolute cross-ratio are defined by

$$
[a, b, c, d]=\frac{(a-c) \cdot(b-d)}{(a-d) \cdot(b-c)}
$$

and

$$
|a, b, c, d|=\frac{|a-c| \cdot|b-d|}{|a-d| \cdot|b-c|}
$$

respectively. Note that $|a, b, c, d|$ is the absolute value of $[a, b, c, d]$, and the absolute cross-ratio is well defined for points in $\mathbb{R}^{n}$, whereas the cross-ratio is not.

We say a map $f: \hat{\mathbb{C}} \rightarrow \hat{\mathbb{C}}$ preserves cross-ratios, or preserves absolute cross-ratios respectively, if for any four distinct points $a, b, c, d \in \hat{\mathbb{C}},[f(a), f(b), f(c), f(d)]=$ $[a, b, c, d]$, or $|f(a), f(b), f(c), f(d)|=|a, b, c, d|$, respectively. Obviously, a Möbius transformation

$$
f(z)=\frac{a_{1} z+a_{2}}{a_{3} z+a_{4}}: \hat{\mathbb{C}} \mapsto \hat{\mathbb{C}} \quad\left(a_{1}, a_{2}, a_{3}, a_{4} \in \mathbb{C}, a_{1} a_{4}-a_{2} a_{3} \neq 0\right)
$$

preserves cross-ratios and absolute cross-ratios. We call

$$
f(z)=\frac{a_{1} \bar{z}+a_{2}}{a_{3} \bar{z}+a_{4}}: \hat{\mathbb{C}} \mapsto \hat{\mathbb{C}} \quad\left(a_{1}, a_{2}, a_{3}, a_{4} \in \mathbb{C}, a_{1} a_{4}-a_{2} a_{3} \neq 0\right)
$$

a conjugate Möbius transformation. It preserves absolute cross-ratios but does not preserve cross-ratios.

In higher dimensions, a Möbius transformation is a homeomorphism acting on $\hat{\mathbb{R}}^{n}$, which is a finite composition of reflections (or inversions) in spheres. A Möbius transformation of $\hat{\mathbb{R}}^{n}$ onto itself preserves absolute cross-ratios.

There are many papers about Möbius invariant or rigidity properties in classes of mappings under various assumptions (see, for example, [1-3,5-16]).

In 1998 Haruki and Rassias [5] obtained the following rigidity result related to the absolute cross-ratios in $\hat{\mathbb{C}}$.

Theorem A [5] Let $D \subset \hat{\mathbb{C}}$ be a domain. Suppose that a map $f: D \rightarrow \hat{\mathbb{C}}$ is meromorphic and satisfies that $|a, b, c, d|=1$ implies $|f(a), f(b), f(c), f(d)|=1$ for every $a, b, c, d \in D$. Then $f$ is a Möbius transformation.

In 2001 Beardon and Minda [2] extended the result related to the absolute crossratios for all dimensions.

Theorem B [2] Suppose that $\Omega \subset \hat{\mathbb{R}}^{n}$ is a region and an injection $f: \Omega \rightarrow \hat{\mathbb{R}}^{n}$ satisfies that $|a, b, c, d|=1$ implies $|f(a), f(b), f(c), f(d)|=1$ for every $a, b, c, d \in \Omega$. Then $f$ is a Möbius transformation. 
In 2014 Yang [15] further extended the results to a more general setting.

Theorem C [15] Let $\Omega_{1}, \Omega_{2}$ be two domains in Hilbert space. Given $k>0$, suppose that a bijection $f: \Omega_{1} \rightarrow \Omega_{2}$ satisfies that for every $a, b, c, d \in \Omega_{1},|a, b, c, d|=k$ implies $|f(a), f(b), f(c), f(d)|=k$. Then $f$ is a Möbius transformation.

In the case of the extended complex plane, Beardon and Minda [2] mentioned that if $f: \hat{\mathbb{C}} \rightarrow \hat{\mathbb{C}}$ preserves all cross-ratios, then it is Möbius, but if $f$ preserves cross-ratios with one value -1 (which means that $[a, b, c, d]=-1$ implies $[f(a), f(b), f(c), f(d)]=-1)$, then $f$ need not be Möbius. They proposed the question: "What can be said about the intermediate situations? More precisely, is it possible to characterize the subsets $E$ of $\mathbb{C}$ with the property that if $[f(a), f(b), f(c), f(d)] \in$ $E$ whenever $[a, b, c, d] \in E$, then $f$ is Möbius?"

Definition 1.1 For a subset $E \subset \hat{\mathbb{C}}$, a map $f: \hat{\mathbb{C}} \rightarrow \hat{\mathbb{C}}$ preserves cross-ratios in $E$, if $[f(a), f(b), f(c), f(d)] \in E$ whenever $[a, b, c, d] \in E$.

We restate their problem as the following.

Problem 1.2 (Beardon and Minda [2]) Is it possible to characterize subsets $E$ of $\mathbb{C}$ with the property that if $f$ preserves cross-ratios in E, then $f$ is Möbius?

We remark that since similar rigidity problems related to absolute cross-ratios are already solved in all dimensions, in the three papers above, the problem related to cross-ratios makes sense only for $\hat{\mathbb{C}}$.

In this paper, we shall give a positive answer to this problem by proving the following two theorems.

Theorem 1.3 Let $E \not \subset \hat{\mathbb{R}}$ be an arc (or circle) and $\bar{E}=\{\bar{z} \mid z \in E\}$. If $f: \hat{\mathbb{C}} \mapsto \hat{\mathbb{C}}$ preserves cross-ratios in $E$, then $f$ is a Möbius transformation when $\bar{E} \neq E$ and $f$ is a Möbius or conjugate Möbius transformation when $\bar{E}=E$.

Theorem 1.4 Suppose that $E \subset \hat{\mathbb{R}}$ is any (open or closed) arc satisfying that the cardinal number $\#(E \cap\{0,1, \infty\})<2$. If a map $f: \hat{\mathbb{C}} \mapsto \hat{\mathbb{C}}$ preserves cross-ratios in E, then $f$ is a Möbius or conjugate Möbius transformation.

Remark 1 The converse is true. If $f$ is a Möbius or conjugate Möbius transformation, then $f$ preserves cross-ratios in $E$.

We next show that the assumptions in our theorems are necessary and reasonable by the following example.

Example 1.5 Since the plane and the real line have the same cardinality, the classic Cantor-Schroeder-Bernstein Theorem [4] guarantees that there exists a bijection $g$ : $\hat{\mathbb{C}} \mapsto \hat{\mathbb{R}}$. Therefore $g$ is injective and circle-into-circle, but $g$ is not a Möbius nor a conjugate Möbius transformation. Obviously, for any four distinct points $a, b, c, d$, $[g(a), g(b), g(c), g(d)] \in \mathbb{R} \backslash\{0,1\}$, as $g(a), g(b), g(c), g(d)$ share a common circle $\hat{\mathbb{R}}$.

For any set $E \subset \hat{\mathbb{C}}$ satisfying that $\mathbb{R} \backslash\{0,1\} \subset E$, the injection $g: \hat{\mathbb{C}} \mapsto \hat{\mathbb{R}}$ always preserves cross-ratios in $E$. Therefore one cannot expect results similar to those in the theorems for such a set $E$ in cross-ratios preserving case. 
Furthermore, if we let $E=\hat{\mathbb{R}}$, then $\#(E \cap\{0,1, \infty\})=3$. Of course if we let $E=\mathbb{R}$, then $\#(E \cap\{0,1, \infty\})=2$. Therefore the assumption that $\#(E \cap\{0,1, \infty\})<2$ in the case $E \subset \hat{\mathbb{R}}$ is necessary.

As a consequence, we have the following.

Corollary 1.6 Let $\theta \in \mathbb{R} \backslash \mathbb{Z}$ and $E^{\theta}=\left\{r e^{i \theta \pi} \in \mathbb{C}: r>0\right\}$. If $f: \hat{\mathbb{C}} \mapsto \hat{\mathbb{C}}$ preserves cross-ratios in $E^{\theta}$, then $f$ is a Möbius transformation. If $\theta \in \mathbb{Z}$ and $f: \hat{\mathbb{C}} \mapsto \hat{\mathbb{C}}$ preserves cross-ratios in $E^{\theta}$, then $f$ is a Möbius or conjugate Möbius transformation.

\section{The Proof of Theorem 1.3}

We need the following two Lemmas.

Lemma 2.1 Let $E$ be an arc (or circle) and $\{0,1, \infty\} \not \subset$ E. If a map $f: \hat{\mathbb{C}} \mapsto \hat{\mathbb{C}}$ preserves cross-ratios in $E$, then $f$ is injective and circle-into-circle.

Proof Without loss of generality, we may suppose that $0 \notin E$ and choose $\mu \in$ $E \backslash\{0,1, \infty\}$. Assume that there exist two distinct points $z_{1}, z_{2}$, such that $f\left(z_{1}\right)=$ $f\left(z_{2}\right)$. Choose $w_{1}, w_{2} \in \hat{\mathbb{C}}$, such that $\left[z_{1}, w_{1}, z_{2}, w_{2}\right]=\mu \in E$. Then we have either $\left[f\left(z_{1}\right), f\left(w_{1}\right), f\left(z_{2}\right), f\left(w_{2}\right)\right]=0 \notin E$ or the cross-ratio is not well defined for $f\left(z_{1}\right)=f\left(z_{2}\right)$. This is a contradiction. Therefore $f$ is injective.

For any four distinct points $a, b, c, d$ sharing a common circle, there exists a Möbius transformation $\sigma$ on $\hat{\mathbb{C}}$, such that $\sigma(E) \ni a, b, c, d$. Let $z_{0}=\sigma(0), z_{1}=\sigma(1)$ and $z_{\infty}=\sigma(\infty)$. Then $\left[z_{0}, z_{\infty}, z, z_{1}\right]=\sigma^{-1}(z)$, since the Möbius transformation $\sigma$ preserves cross-ratios.

For any $z \in \sigma(E), \sigma^{-1}(z) \in E$ and $\left[z_{0}, z_{\infty}, z, z_{1}\right] \in E$. Since $f$ preserves cross-ratios in $E$, we have $\left[f\left(z_{0}\right), f\left(z_{\infty}\right), f(z), f\left(z_{1}\right)\right] \in E$. On the other hand, let $\tau$ be the Möbius transformation mapping $0,1, \infty$ to $f\left(z_{0}\right), f\left(z_{1}\right), f\left(z_{\infty}\right)$. Then $\left[f\left(z_{0}\right), f\left(z_{\infty}\right), f(z), f\left(z_{1}\right)\right]=\tau^{-1}(f(z))$, which means that $f(z)$ lies in the arc $\tau(E)$ for any $z \in \sigma(E)$ and $f(a), f(b), f(c), f(d)$ share a common circle, from which we conclude that $f$ is circle-into-circle and this completes the proof.

Lemma $2.2[10]$ Suppose that $f: \hat{\mathbb{C}} \mapsto \hat{\mathbb{C}}$ is injective and circle-into-circle. If $f(\hat{\mathbb{C}})$ does not lie on a circle, then $f$ is a Möbius or conjugate Möbius transformation.

Proof of Theorem 1.3 Let $E \not \subset \hat{\mathbb{R}}$ be an arc (or circle). If $f$ preserves crossratios in $E$, then $f$ is injective and circle-into-circle by Lemma 2.1. So, for any $z \in E,[f(0), f(\infty), f(z), f(1)] \in E$ as $[0, \infty, z, 1]=z \in E$. Moreover, for any two distinct points $z_{1}, z_{2} \in E,\left[f(0), f(\infty), f\left(z_{1}\right), f(1)\right] \neq$ $\left[f(0), f(\infty), f\left(z_{2}\right), f(1)\right]$, since $f$ is injective. There exists a point $z_{0} \in E$, such that $\left[f(0), f(\infty), f\left(z_{0}\right), f(1)\right] \notin \hat{\mathbb{R}}$ by $\#(E \cap \hat{\mathbb{R}})<3$. That is, $f(0), f(\infty), f\left(z_{0}\right)$, $f(1)$ do not lie in a common circle. Then $f$ is a Möbius or conjugate Möbius transformation by Lemma 2.2.

In particular, if $\bar{E}=E$ and $f$ preserves cross-ratios in $E$, then $f$ is a Möbius or conjugate Möbius transformation.

If $\bar{E} \neq E$, then there exist $\mu \in E \backslash \bar{E}$ and four distinct points $a, b, c, d$ such that $[a, b, c, d]=\mu$. We show that $f$ is a Möbius transformation. On the contrary 
assume that $f$ is a conjugate Möbius transformation. Then $\bar{f}(z)$ is a Möbius transformation and $[\bar{f}(a), \bar{f}(b), \bar{f}(c), \bar{f}(d)]=\mu$. So $[f(a), f(b), f(c), f(d)]=\bar{\mu} \notin E$. This is a contradiction, since $f$ preserves cross-ratios in $E$. Therefore $f$ is a Möbius transformation. The proof is completed.

\section{The Proof of Theorem 1.4}

Suppose that an arc $E \subset \hat{\mathbb{R}}$ satisfies that $\{0,1, \infty\} \not \subset E$ and a map $f: \hat{\mathbb{C}} \mapsto \hat{\mathbb{C}}$ preserves cross-ratios in $E$. Then $f$ is injective and circle-into-circle by Lemma 2.1. Note that $f$ is a Möbius or conjugate Möbius transformation if and only if $f(\hat{\mathbb{C}})$ does not lie in a circle by Lemma 2.2. Example 1.5 shows that there exists some injective and circle-into-circle map whose image lie in a circle. In this section, we shall prove that if $\#(E \cap\{0,1, \infty\})<2$ and $f$ preserves cross-ratios in $E$, then $f$ is a Möbius or conjugate Möbius transformation.

For the sake of convenience, let $\Gamma_{(a, b, c)}$ denote the circle determined by $a, b, c \in \hat{\mathbb{C}}$ and $\gamma_{[a, b]}^{c}$ denote the arc in $\Gamma_{(a, b, c)}$ with endpoints $a, b$ and containing $c$. Denote the line segment with endpoints $a, b \in \mathbb{C}$ by $I_{[a, b]}$. Similarly, let $I_{(a, b)}\left(I_{[a, b)}\right.$ or $\left.I_{(a, b]}\right)$ denote an (sub-)open segment, and $\gamma_{(a, b)}^{c}\left(\gamma_{[a, b)}^{c}\right.$ or $\left.\gamma_{(a, b]}^{c}\right)$ denote an (sub-)open arc. Obviously, if $\#(E \cap\{0,1, \infty\})<2$, then $E$ must be a sub-arc of either $\gamma_{(0, \infty)}^{1}$, or $\gamma_{(1, \infty)}^{0}$, or $\gamma_{(0,1)}^{\infty}$. We shall prove Theorem 1.4 for the case of $E \subset \gamma_{(0, \infty)}^{1}$. For the other two cases, the discussions are similar and so we shall omit the details.

We need the following lemmas.

Lemma 3.1 For any $a, b, c, d \in \hat{\mathbb{C}},[a, b, c, d] \in \gamma_{(0, \infty)}^{1}$ if and only if $c \in \gamma_{(a, b)}^{d}$.

Proof Suppose that $\sigma$ is the Möbius transformation such that $\sigma(a)=0, \sigma(b)=\infty$ and $\sigma(d)=1$. Then $[a, b, c, d]=\sigma(c) \in \gamma_{(0, \infty)}^{1}$, which means $c \in \gamma_{(a, b)}^{d}$.

Lemma 3.2 Suppose that $E \subset \gamma_{(0, \infty)}^{1}$ is an arc and a map $f: \hat{\mathbb{C}} \mapsto \hat{\mathbb{C}}$ preserves cross-ratios in E. Then $f$ maps $\gamma_{[a, b]}^{d}$ into $\gamma_{[f(a), f(b)]}^{f(d)}$ for any three distinct points $a, b, d \in \hat{\mathbb{C}}$.

Proof For any $z_{0} \in \gamma_{(a, b)}^{d}$, we prove that $f\left(z_{0}\right) \in \gamma_{(f(a), f(b))}^{f(d)}$. Let $I$ be an open segment in $E$ containing some point $p_{0}$. Let $\sigma$ be the Möbius transformation mapping $a, b, d$ to $0, \infty, 1$ respectively. Denote $c_{0}=\sigma^{-1}\left(p_{0}\right)$. For any $z \in \sigma^{-1}(E),[a, b, z, d]=$ $\sigma(z) \in E$. So $[f(a), f(b), f(z), f(d)] \in E$ and $f(z) \in \gamma_{(f(a), f(b))}^{f(d)}$.

Let $J$ be the closed arc in $\gamma_{(a, b)}^{d}$ with endpoints $c_{0}, z_{0}$. For any $z \in J$, there exists a Möbius transformation $\sigma_{z}$ which maps $a, b, z$ to $0, \infty, p_{0}$. Obviously, $\left\{\sigma_{z}^{-1}(I)\right\}_{z \in J}$ is an open covering of the closed segment $J$, which contains a finite sub-covering. That is, there is an integer $k>0$, and $z_{1}, z_{2}, \ldots, z_{k} \in J$, such that $\left\{\sigma_{z_{i}}^{-1}(I)\right\}_{i=1}^{k}$ is an open covering of $J$. So $\left\{\sigma_{z_{i}}^{-1}(E)\right\}_{i=1}^{k}$ covers $J$. Let $d_{i}=\sigma_{z_{i}}^{-1}(1)$. For any $i$ and $z \in \sigma_{z_{i}}^{-1}(E)$, $\left[a, b, z, d_{i}\right] \in E$ and $\left[f(a), f(b), f(z), f\left(d_{i}\right)\right] \in E$, which means $f(z) \in \gamma_{[f(a), f(b)]}^{f\left(d_{i}\right)}$ by Lemma 3.1. Therefore $f\left(z_{0}\right) \in \gamma_{[f(a), f(b)]}^{f(d)}$. 
Remark 2 Similarly, $[a, b, c, d] \in \gamma_{(1, \infty)}^{0}$ implies $c \in \gamma_{(b, d)}^{a}$ and $[a, b, c, d] \in \gamma_{(0,1)}^{\infty}$ implies $c \in \gamma_{(a, d)}^{b}$. If $f$ preserves cross-ratios in $E \subset \gamma_{(1, \infty)}^{0}$, then $f$ maps $\gamma_{[b, d]}^{a}$ into $\gamma_{[f(b), f(d)]}^{f(a)}$. If $f$ preserves cross-ratios in $E \subset \gamma_{(0,1)}^{\infty}$, then $f$ maps $\gamma_{[a, d]}^{b}$ into $\gamma_{[f(a), f(d)]}^{f(b)}$.

Remark 3 Let $E^{\circ}$ be the set of inner points of $E$. The condition $\#(E \cap\{0,1, \infty\})<2$ in Theorem 1.4 can be replaced by $\#(E \cap\{0,1, \infty\})<3$ and $\#\left(E^{\circ} \cap\{0,1, \infty\}\right)<2$. For example, if $E \subset \gamma_{[0, \infty)}^{1}$, then $[a, b, c, d] \in E$ implies $c \in \gamma_{[a, b)}^{d}$.

Definition 3.3 A circle-into-circle injection $f$ is separation-preserving in circle, if, for any circle $\gamma$ and four distinct points $a, b, c, d \in \gamma, a, b$ separate $c$ and $d$ in $\gamma$ implies that $f(a), f(b)$ separate $f(c)$ and $f(d)$ in a circle $\Gamma \supset f(\gamma)$.

Lemma 3.4 An injection $f: \hat{\mathbb{C}} \mapsto \hat{\mathbb{C}}$ maps $\gamma_{[a, b]}^{d}$ into $\gamma_{[f(a), f(b)]}^{f(d)}$ for any three distinct points $a, b, d \in \hat{\mathbb{C}}$, if and only if $f$ is separation-preserving in circle.

Proof Assume $f$ is separation-preserving in circle. That is, for any four distinct points $a, b, c, d$ in a common circle satisfying that $a, b$ separate $c$ and $d, f(a), f(b)$ separate $f(c)$ and $f(d)$. Then for any $z \in \gamma_{(a, b)}^{d}, a, b$ separate $c$ and $z$ and $f(a), f(b)$ separate $f(c)$ and $f(z)$, which implies that $f(z) \in \gamma_{(f(a), f(b))}^{f(d)}$ for any three distinct points $a, b, d \in \hat{\mathbb{C}}$.

Assume $f$ is not separation-preserving in circle. That is, there exist four distinct points $a, b, c, d$ in a common circle satisfying that $a, b$ separate $c$ and $d$, such that $f(a), f(b)$ do not separate $f(c)$ and $f(d)$. Assume without loss of generality that $f(b)$, $f(d)$ separate $f(a)$ and $f(c)$. Then we find that $a \in \gamma_{(b, d)}^{c}$ and $f(a) \notin \gamma_{(f(b), f(d))}^{f(c)}$. That is, $f$ does not map $\gamma_{[b, d]}^{c}$ into $\gamma_{[f(b), f(d)]}^{f(c)}$. This completes the proof.

From Lemmas 2.1, 3.2 and 3.4, we have the following lemma.

Lemma 3.5 Suppose that $E \subset \hat{\mathbb{R}}$ is an arc satisfying that $\#(E \cap\{0,1, \infty\})<2$. If $f: \hat{\mathbb{C}} \mapsto \hat{\mathbb{C}}$ preserves cross-ratios in $E$, then $f$ is separation-preserving in circle.

Let $\operatorname{Im}(z)$ and $\operatorname{Re}(z)$ denote the imaginary and real part of $z \in \mathbb{C}$ respectively. Denote the upper half plane of $\mathbb{C}$ by $H=\{z \in \mathbb{C}: \operatorname{Im}(z)>0\}$ and the lower one by $\Omega$. To obtain Theorem 1.4, we consider the case where $f(\hat{\mathbb{C}})$ lies in a circle.

Lemma 3.6 Suppose that a map $f: \hat{\mathbb{C}} \mapsto \hat{\mathbb{R}}$ is separation-preserving in circle and fixes $1,-1, \infty$. If there exists $z_{0} \in H$ such that $f\left(z_{0}\right) \in I_{(-1,1)}$, then $f(H) \subset I_{(-1,1)}$.

Proof Note that $f(z) \subset I_{(-1,1)}$ for any $z \in \gamma_{(-1,1)}^{z_{0}} \cup I_{(-1,1)}$. Denote the bounded component of $\hat{\mathbb{C}} \backslash\left(I_{[-1,1]} \cup \gamma_{[-1,1]}^{z_{0}}\right)$ by $D_{1}$. We claim that $f(z) \subset I_{(-1,1)}$ for any $z \in D_{1}$. Let $u$ be the point of intersection of $\gamma_{(-1, \infty)}^{z}$ and $\gamma_{(-1,1)}^{z_{0}}$. So $f(z) \in I_{(-1, f(u))} \subset I_{(-1,1)}$ (see Fig. 1).

Let $z_{1}, w_{1}$ be points in $\Gamma_{\left(-1,1, z_{0}\right)}$ satisfying that $\operatorname{Re}\left(z_{1}\right)=\operatorname{Re}\left(w_{1}\right)=0, \operatorname{Im}\left(z_{1}\right)>0$ and $\operatorname{Im}\left(w_{1}\right)<0$, and $o_{1}=\left(z_{1}+w_{1}\right) / 2$ be the center of $\Gamma_{\left(-1,1, z_{0}\right)}$. Let (see Fig. 2)

$$
o_{n+1}=\frac{z_{n}+o_{n}}{2}, \quad z_{n+1}=o_{n+1}+i \sqrt{1+\operatorname{Im}\left(o_{n+1}\right)^{2}}
$$




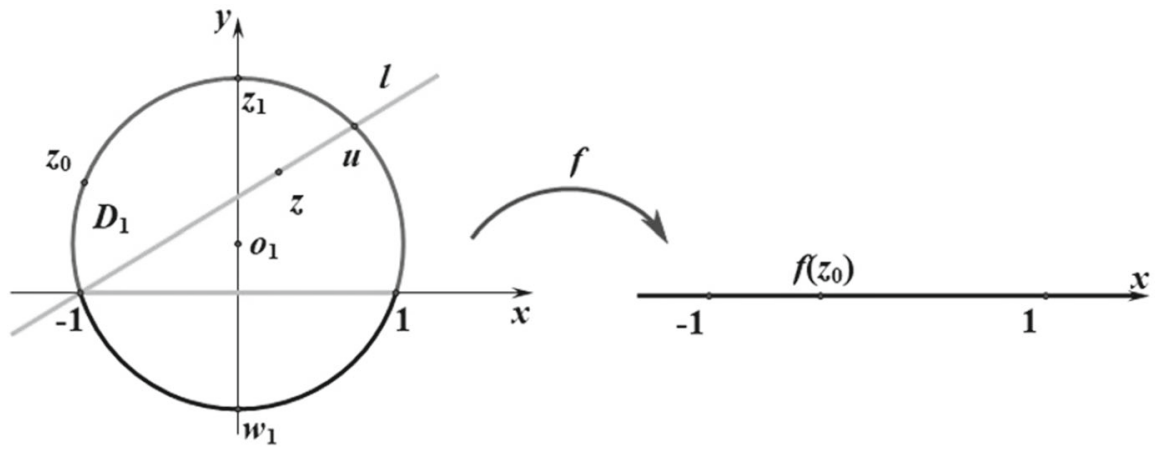

Fig. $1 f\left(D_{1}\right) \subset I_{(-1,1)}$

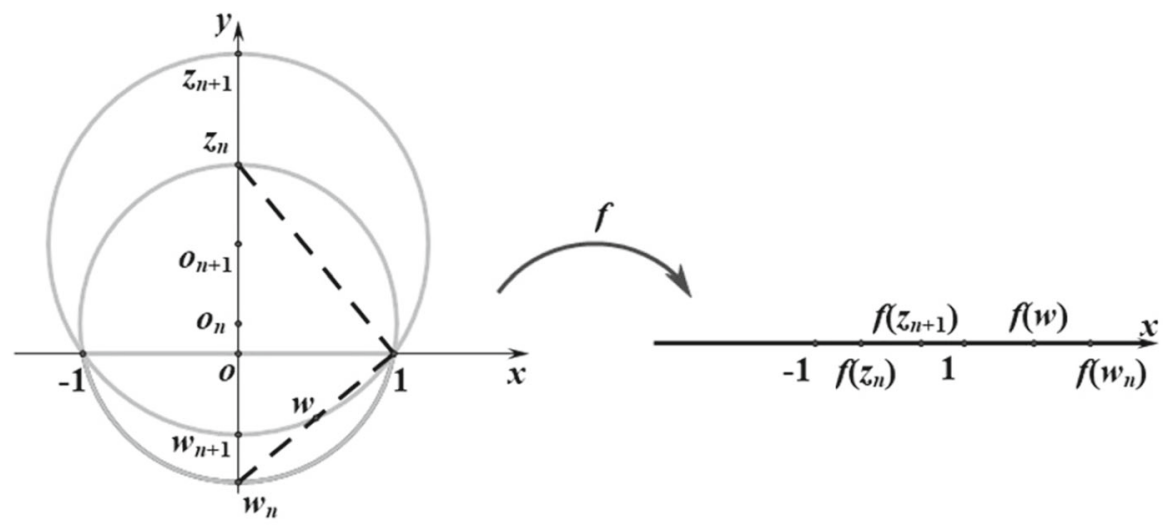

Fig. $2 f(H) \subset I_{(-1,1)}$

and $w_{n+1}=2 o_{n+1}-z_{n+1}$, for $n=1,2, \ldots$ Obviously, $o_{n}$ is the centre of $\Gamma_{\left(-1,1, z_{n}\right)}$, and $z_{n}, w_{n} \in \Gamma_{\left(-1,1, z_{n}\right)}$ satisfy $\operatorname{Re}\left(z_{n}\right)=\operatorname{Re}\left(w_{n}\right)=0, \operatorname{Im}\left(z_{n}\right)>0$ and $\operatorname{Im}\left(w_{n}\right)<0$ for any $n \geq 1$. We have

$$
o_{n+1}=o_{n}+\frac{i}{2} \sqrt{1+\operatorname{Im}\left(o_{n}\right)^{2}}, \quad \operatorname{Re}\left(o_{n}\right)=0
$$

and the sequence $\left\{\operatorname{Im}\left(o_{n}\right)\right\}_{n=1}^{\infty}$ strictly increases. Then $\operatorname{Im}\left(o_{n}\right)$ tends to $+\infty$ as $n$ tends to $+\infty$, which is due to the fact that $\operatorname{Im}\left(o_{n+1}\right)-\operatorname{Im}\left(o_{n}\right) \geq 1 / 2$ for any $n \geq 1$. Therefore $\operatorname{Im}\left(z_{n}\right) \rightarrow+\infty$ and $\operatorname{Im}\left(w_{n}\right)=-1 / \operatorname{Im}\left(z_{n}\right) \rightarrow 0$ as $n$ tends to $+\infty$.

We shall prove that $f\left(z_{n}\right) \in I_{(-1,1)}$ for all $n \geq 1$ by induction. As $f\left(z_{1}\right) \in I_{(-1,1)}$, we may suppose that $f\left(z_{n}\right) \in I_{(-1,1)}$ for some $n$. Since $f$ is separation-preserving in circle, $f\left(w_{n}\right) \notin I_{[-1,1]}$. If $f\left(w_{n}\right)>1$ (see Fig. 2), let $w$ be the point of intersection

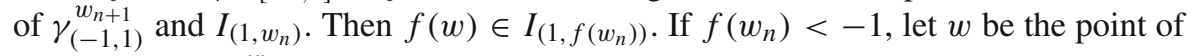
intersection of $\gamma_{(-1,1)}^{w_{n+1}}$ and $I_{\left(w_{n},-1\right)}$. Then $f(w) \in I_{\left(f\left(w_{n}\right),-1\right)}$. There always exists a point $w \in \gamma_{(-1,1)}^{w_{n+1}}$, such that $f(w) \notin I_{[-1,1]}$. Then $f\left(z_{n+1}\right) \in I_{(-1,1)}$ since $z_{n+1}$ is separated with $w$ by $-1,1$ in the circle $\Gamma_{\left(-1,1, z_{n+1}\right)}$. 


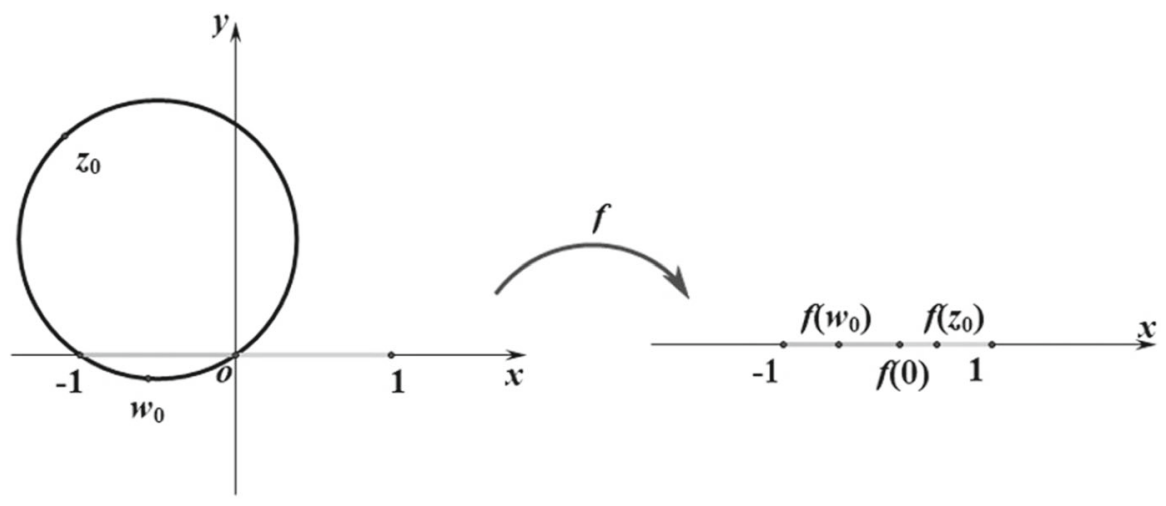

Fig. 3 Contradiction: $\exists w_{0} \in \Omega$, such that $f\left(w_{0}\right) \in I_{(-1,1)}$

Denote the bounded component of $\hat{\mathbb{C}} \backslash\left(I_{[-1,1]} \cup \gamma_{[-1,1]}^{z_{n}}\right)$ by $D_{n}$. Then $f\left(D_{n}\right) \subset$ $I_{(-1,1)}$ for $f\left(z_{n}\right) \in I_{(-1,1)}$ for any $n$, which means $f(H) \subset I_{(-1,1)}$.

Proof of Theorem 1.4 It follows from Lemma 3.5 that $f$ is separation-preserving in circle. We shall prove that $f(\hat{\mathbb{C}})$ does not lie in a circle. To the contrary we assume that $f$ maps $\hat{\mathbb{C}}$ into $\hat{\mathbb{R}}$ and fixes $1,-1$ and $\infty$ by composing some suitable Möbius transformations. Moreover, there always exists $z_{0} \in H$, such that $f\left(z_{0}\right) \subset I_{(-1,1)}$, since we may replace $f(z)$ by $f(\bar{z})$, if necessary. Lemma 3.6 gives that $f(z) \in I_{(-1,1)}$ for any $z \in H$.

Without loss of generality, we may assume that $f\left(z_{0}\right) \in I_{(f(0), 1)}$. Let $w_{0}$ be a point in $\Gamma_{\left(-1,0, z_{0}\right)} \cap \Omega$. Then $f\left(z_{0}\right) \notin I_{[-1, f(0)]}$ (see Fig. 3), which means that $f\left(w_{0}\right) \in$ $I_{(-1, f(0))} \subset I_{(-1,1)}$.

Again we have from Lemma 3.6 that $f(w) \in I_{(-1,1)}$ for any $w \in \Omega$. Meanwhile, $w_{n} \in \Omega$ and $f\left(w_{n}\right) \notin I_{(-1,1)}$ for any $n \geq 1$ as in Lemma 3.6. This is a contradiction. Therefore $f(\hat{\mathbb{C}})$ does not lie in a circle. It then follows from Lemma 2.2 that $f$ is a Möbius or conjugate Möbius transformation. The proof is completed.

Acknowledgements The authors would like to express their gratitude to the referees for their careful reading over the manuscript and for giving valuable suggestions and comments, which were very helpful in improving the paper.

Open Access This article is licensed under a Creative Commons Attribution 4.0 International License, which permits use, sharing, adaptation, distribution and reproduction in any medium or format, as long as you give appropriate credit to the original author(s) and the source, provide a link to the Creative Commons licence, and indicate if changes were made. The images or other third party material in this article are included in the article's Creative Commons licence, unless indicated otherwise in a credit line to the material. If material is not included in the article's Creative Commons licence and your intended use is not permitted by statutory regulation or exceeds the permitted use, you will need to obtain permission directly from the copyright holder. To view a copy of this licence, visit http://creativecommons.org/licenses/by/4.0/. 


\section{References}

1. Aczél, J., McKiernan, M.: On the characterization of plane projective and complex Möbius transformations. Math. Nachr. 33, 315-337 (1967)

2. Beardon, A., Minda, D.: Sphere-preserving maps in inversive geometry. Proc. Am. Math. Soc. 130(4), 987-998 (2001)

3. Carathéodory, C.: The most general transformations of plane regions which transform circles into citcles. Bull. Am. Math. Soc. 43, 573-579 (1937)

4. Fraenkel, A.: Abstract Set Theory. North-Holland, Amsterdam (1953)

5. Haruki, H., Rassias, T.: A new characteristic of Möbius transformations by use of Apollonius quadrilaterals. Proc. Am. Math. Soc. 126(10), 2857-2861 (1998)

6. Höfer, R.: A characterization of Möbius transformations. Proc. Am. Math. Soc. 128(4), 1197-1201 (1999)

7. Jeffers, J.: Lost theorems of geometry. Am. Math. Mon. 107(9), 800-812 (2000)

8. Li, B., Wang, X., Wang, Y.: The pseudo-affine transformations in $\mathbb{R}^{2}$. Sci. China Math. 53(3), 755-762 (2010)

9. Li, B., Wang, Y.: Transformations and non-degenerate maps. Sci. China Math. 48, 195-205 (2005)

10. Li, B., Wang, Y.: Fundamental theorem of geometry without surjective assumption. Trans. Am. Math. Soc. 368(10), 6819-6834 (2016)

11. Li, B., Wang, Y.: A new characterization of line-to-line maps in the upper plane. Filomat 27, 127-133 (2013)

12. Li, B., Yao, G.: On characterizations of sphere-preserving maps. Math. Proc. Camb. Philos. Soc. 147, 439-446 (2009)

13. Nehari, Z.: Conformal Mapping. McGraw-Hill, New York (1952)

14. Özgür, N., Bulut, S.: A note on the characteristic properties of Möbius transformations. Rad. Mat. 12, 129-133 (2004)

15. Yang, S.: On the properties of sphere-preserving maps. J. Math. Anal. Appl. 419, 748-755 (2014)

16. Yao, G.: Transformations of spheres without the injectivity assumption, Quasiconformal mappings, Riemann surfaces, and Teichmüller spaces, pp. 371-375, Contemp. Math., vol. 575, Amer. Math. Soc., Providence (2012)

Publisher's Note Springer Nature remains neutral with regard to jurisdictional claims in published maps and institutional affiliations. 